\title{
Efeitos da cesárea eletiva sobre desfechos no primeiro ano de vida: estudo de coorte
}

\author{
Effects of elective cesarean section on outcomes in the first year of life: a cohort study \\ Efectos de la cesárea electiva sobre los resultados en el primer año de vida: un estudio de cohort
}

\author{
Anna Paula Ferrari ${ }^{1}$ (D) \\ Maria Cristina Heinzle da Silva Machado ${ }^{1}$ (1) \\ Vera Lúcia Pamplona Tonete ${ }^{1}$ (1) \\ Cristina Maria Garcia de Lima Parada ${ }^{1}$ (D)
}

1. Universidade Estadual Paulista "Júlio de Mesquita Filho", Faculdade de Medicina de Botucatu. Botucatu, SP, Brasil.
Autor correspondente: Anna Paula Ferrari.

E-mail: anna_ferrari04@yahoo.com.br.

Recebido em 29/07/2020.

Aprovado em 23/12/2020.

DOI:https://doi.org/10.1590/2177-9465-EAN-2020-0273

\section{Resumo}

Objetivo: verificar o efeito da cesárea eletiva, em comparação ao parto vaginal, sobre os desfechos no primeiro ano de vida da criança. Método: estudo de coorte com 499 mães e seus bebês. Foram coletados dados sociodemográficos, relativos à história gestacional, ao parto e nascimento e sobre o primeiro ano de vida do bebê, em entrevista realizada na unidade de triagem neonatal, do registro do prontuário da maternidade e em entrevistas no domicílio. Avaliaram-se as associações de interesse mediante análises de regressão de Cox, ajustadas para as covariáveis identificadas, considerando-se resultados de análises bivariadas que apresentaram significância estatística em nível de $p<0,20$. Nas análises ajustadas, consideraram-se as relações significativas se $\mathrm{p}<0,05$, tendo como medida de efeito o risco relativo. Resultados: as cesáreas eletivas não se associaram aos desfechos estudados (interrupção do aleitamento materno, infecções respiratórias, atopias e sobrepeso/obesidade). Conclusão e implicações para a prática: a ausência de associação da cesárea eletiva e os desfechos do primeiro ano de vida poderão ser confirmados em investigações futuras. Pelos achados, sugere-se que a equipe de saúde apoie as mães, com o intuito de aumentar sua confiança e empenho em relação à amamentação, prática que pode repercutir positivamente nos demais desfechos estudados.

Palavras-chave: Parto; Cesárea; Procedimentos Cirúrgicos Eletivos; Morbidade; Lactente.

\section{Abstract}

Objective: to verify the effect of elective cesarean section compared to vaginal delivery, on the outcomes of the child's first year of life. Methods: cohort study with 499 mothers and their babies. Sociodemographic data were collected, related to the gestational history, birth and delivery, and about the baby's first year of life, in an interview carried out at the neonatal screening unit, from the maternity records and in home interviews. The associations of interest were evaluated with Cox regression analyses adjusted for the identified covariates, considering results of bivariate analyses that presented statistical significance at a $p<0.20$ level. In the adjusted analyses, associations were considered significant if $p<0.05$, having as effect measure the relative risk. Results: elective cesarean sections were not associated with the outcomes studied (interruption of breastfeeding, respiratory infections, atopies and overweight/obesity). Conclusion and implications for practice: the absence of association of elective cesarean sections and first year outcomes may be confirmed in future investigations. Based on the findings, it is suggested that the health team should support mothers in order to increase their confidence and commitment to breastfeeding, a practice that may have a positive impact on the other outcomes studied.

Keywords: Childbirth; Cesarean section; Elective Surgical Procedures; Morbidity; Infant.

\section{Resumen}

Objetivo: verificar el efecto de cesárea electiva, comparándolo con el producido por parto vaginal, en los resultados del primer año de vida del niño. Métodos: estudio de cohorte en 499 madres y sus bebés. Fueron recogidos datos sociodemográficos relacionados a la historia gestacional, al parto, al nacimiento y al primer año de vida del bebé; los datos fueron obtenidos en entrevista realizada en la unidad de selección neonatal, en la ficha médica de la maternidad y en entrevistas en el domicilio. Las asociaciones fueron evaluadas con el análisis de regresión Cox, ajustadas para las covariables identificadas, considerando los resultados del análisis bivariado que presentaron significación estadística con un nivel de $p<0,20$. En los análisis ajustados, las relaciones fueron consideradas significativas si $p<0,05$, teniendo como medida de efecto el riesgo relativo. Resultados: las cesáreas electivas no se asociaron con los resultados estudiados (interrupción del amamantamiento materno, infecciones respiratorias, atopias y exceso de peso/obesidad). Conclusión e implicaciones para la práctica: la ausencia de asociación de la cesárea electiva con los resultados del primer año de vida podrá ser confirmada en investigaciones futuras. De acuerdo con los resultados, se sugiere que el equipo de salud apoye a las madres con la finalidad de aumentar su confianza y empeño para realizar el amamantamiento; esta práctica puede repercutir positivamente en los demás resultados estudiados.

Palabras clave: Parto; Cesárea; Procedimientos Quirúrgicos Electivos; Morbilidad. Lactante. 


\section{INTRODUÇÃO}

Além dos possíveis desfechos adversos observados no parto, ${ }^{1-4}$ a literatura científica tem apontado efeitos deletérios da operação cesariana na primeira infância, incluindo desmame precoce, infecções do trato respiratório, atopias e obesidade. .,6 $^{5}$ Para os desfechos desmame e infecções respiratórias, há distinção entre cesárea eletiva e indicada, ${ }^{7,8}$ o que não ocorre quando se consideram alergia e obesidade.

Revisão sistemática com metanálise, publicada em 2012, evidenciou que a taxa de aleitamento materno com início precoce foi mais baixa entre mulheres submetidas a cesárea, em comparação ao parto vaginal, sendo essa redução ainda mais relevante no caso de cesárea eletiva. ${ }^{9}$ Em estudo prospectivo desenvolvido em Calgary, Canadá, com seguimento até o quarto mês de vida dos bebês, após controlar as características sociodemográficas e do parto, encontrou-se associação entre cesárea eletiva e desmame precoce. Explica-se essa associação pelo fato de a cesárea, ao ser realizada antes do início do trabalho de parto, interferir na liberação de ocitocina e, consequentemente, na lactogênese, dificultando o início e continuidade da amamentação. Assim, o resultado desfavorável acontece tanto entre mulheres que tiveram cesáreas indicadas quanto no caso das cesáreas como procedimentos cirúrgicos eletivos, quando ambos ocorrem antes do início do trabalho de parto. ${ }^{7}$

Sobre as infecções do trato respiratório, ensaio clínico randomizado, desenvolvido por pesquisadores dos Estados Unidos da América, apontou que o nascimento por cesárea resulta em alta morbidade dessas infecções na infância. ${ }^{10}$ Especificamente quanto à ocorrência de asma nessa fase da vida, a literatura científica apresenta resultados ainda divergentes. Em estudo de coorte realizado na Dinamarca, houve associação entre cesárea e asma em crianças maiores de 6 anos, sendo essa associação mais forte em casos de cesárea antes da rotura das membranas. ${ }^{11}$ Porém, estudo com desenho semelhante desenvolvido em Braga, Portugal, envolvendo crianças de 2 anos de idade, não revelou associação entre asma e cesárea eletiva, quando comparadas às nascidas de parto vaginal. ${ }^{8}$

A relação entre atopia e cesárea foi investigada em estudo de coorte realizado na Grécia, que encontrou risco aumentado de desenvolvimento de alergia alimentar na infância em crianças nascidas de cesárea, não sendo associada, porém, à dermatite atópica. ${ }^{12}$ Ao ser privado da passagem pelo canal do parto e, consequentemente, da exposição à microbiota materna, o neonato sofre prejuízo em sua imunidade, o que explica tanto a maior predisposição às atopias quanto às infecções respiratórias. ${ }^{8,10}$

Nesse mesmo sentido, estudo de coorte desenvolvido no Canadá revelou que a microbiota intestinal de crianças nascidas de parto vaginal é mais adequada e diminui o risco de sobrepeso e obesidade na infância. ${ }^{13}$ Estudo similar, ocorrido em Copenhagen, Dinamarca, apontou que, aos 6 meses de vida, bebês nascidos por cesárea tinham velocidade de ganho de peso maior que os nascidos por parto vaginal; no entanto, essa diferença não se manteve na infância. ${ }^{5}$ No Brasil, dois estudos transversais, realizados em estados da região Nordeste, encontraram associação entre excesso de peso na infância ${ }^{14}$ ou em pré-escolare ${ }^{15}$ e parto cesárea. Por outro lado, na região Sul do país, estudo de coorte que avaliou índice de massa corpórea, peso e estatura para idade, concluiu que nascer por cesárea não levou a aumento no risco de obesidade infantil. ${ }^{16}$

Considerando-se a lacuna na literatura em relação aos efeitos deletérios da cesárea eletiva na infância, a escassez de estudos sobre esses efeitos - especificamente no primeiro ano de vida - e as divergências entre os estudos existentes, propõe-se o presente estudo com a hipótese: o efeito da cesárea eletiva é negativo para a criança no primeiro ano de vida, quando comparado ao efeito do parto vaginal, ao considerar desfechos selecionados.

Dessa forma, o objetivo consiste em verificar o efeito da cesárea eletiva, em comparação ao parto vaginal, sobre os desfechos no primeiro ano de vida da criança.

\section{MÉTODO}

Trata-se de estudo de coorte, que integra o estudo CLaB (Coorte de Lactentes de Botucatu), ampla pesquisa financiada pela Fundação de Amparo à Pesquisa do Estado de São Paulo - FAPESP (Processo n. 2015/03256-1), intitulada "Saúde da criança no primeiro ano de vida: estudo de coorte prospectiva no interior paulista".

O município de Botucatu situa-se no estado de São Paulo, região Centro-Sul, com população estimada de 144.820 habitantes para o ano de 2018, e faz parte do Departamento Regional de Saúde VI (DRS VI), Bauru, ao lado de outros 67 municípios. ${ }^{17,18}$

Quanto à assistência ao parto no Sistema Único de Saúde (SUS), no período de coleta de dados, o município contava com um Hospital-Escola e, para assistência às gestantes conveniadas e particulares, um Hospital Privado. ${ }^{18}$ No tocante ao atendimento na primeira semana de vida, além de Unidades Básicas de Saúde de modelo tradicional e da Estratégia Saúde da Família, há no município uma unidade de atenção primária de triagem neonatal, cuja finalidade é assistir a todas as crianças nascidas, tanto no serviço público quanto no privado, e que tem cobertura elevada, acima de $90 \%$ dos partos.

Compuseram a amostra da pesquisa original todos os binômios mãe/bebê que passaram por consulta médica ou de enfermagem na unidade de triagem neonatal, local de captação da coorte, no período definido para o recrutamento - julho de 2015 a fevereiro de 2016. Os critérios de inclusão foram: nascer em uma das duas maternidades de Botucatu; ter no máximo 30 dias de nascido; residir na zona urbana do município; e a mãe ter disponibilidade em receber as entrevistadoras no domicílio, para coleta de dados. Nesse o projeto foi apresentado às mães que levaram seus filhos para o primeiro atendimento clínico após sentido, o nascimento.

O acompanhamento dos bebês ocorreu de julho de 2015 a fevereiro de 2017, quando o último bebê recrutado para o estudo completou 1 ano de vida. No total, 656 bebês (650 mães) foram captados para coorte e 585 foram seguidos até o 12.ํำ mês de vida, ocorrendo $10,8 \%$ de perdas de seguimento. As perdas e 
recusas foram maiores na entrevista em domicílio, aos 6 meses do bebê ( 20 perdas e 6 recusas). Considerou-se perda quando não houve sucesso após três tentativas, em horários diferentes, de contato domiciliar. A recusa ocorreu por manifestação materna em interromper a participação no estudo.

Foram incluídos no grupo cesárea eletiva todos os recémnascidos sem referência a qualquer indicação de parto cirúrgico no prontuário hospitalar. Consideraram-se as seguintes indicações de cesárea: apresentação pélvica; placenta prévia; placenta baixa ou acreta; mãe infectada pelo HIV a depender da carga viral; iteratividade; e herpes genital com lesão ativa. ${ }^{19}$

Excluíram-se, do presente estudo, 6 casos de gemelares (12 bebês), 43 nascidos por cesárea indicada e 31 casos com dados incompletos (missing data). Assim, 499 bebês compuseram a amostra. Porém, considerou-se, para a análise de sobrepeso/ obesidade relacionada ao tipo de parto, porém amostra de 418 crianças (sendo 216 nascidas de parto vaginal e 202 de cesárea eletiva), pois optou-se por incluir apenas aquelas classificadas como eutróficas ao nascimento.

A coleta de dados foi realizada em sete avaliações, por equipe devidamente capacitada, remunerada e supervisionada pela primeira autora deste artigo.

Para responder ao objetivo desta pesquisa foram utilizados dados coletados do primeiro ao sétimo momento de contato com o binômio. Elencam-se, a seguir, as informações coletadas em cada momento do seguimento:

Avaliação 1 - dados de identificação do binômio e referentes à história gestacional atual (coletados por meio de entrevista, na captação); ao parto e ao recém-nascido (coletados do prontuário da maternidade);

Avaliações 2 e 4 - dados coletados sobre aleitamento materno por entrevista telefônica, no segundo e quarto meses de vida do bebê, respectivamente;

Avaliações 3 e 5 a 7 - dados coletados sobre aleitamento materno, infecção respiratória e/ou atopias diagnosticadas e crescimento (peso e comprimento) por entrevista no domicílio, no $3 . \circ, 6 .^{\circ}, 9 .^{\circ}$ e $12 .$. meses de vida da criança.

Para garantir a consistência interna do estudo, entrevistadoras e digitadoras devidamente capacitadas e supervisionadas compuseram a equipe de coleta e de digitação dos dados. Fizeram parte da equipe: 1 supervisora geral de campo, 1 supervisora da coleta e revisores de consistência dos dados, totalizando 22 pessoas envolvidas com a pesquisa. Ademais, contou também com auxílio técnico e logístico de Unidade de Pesquisa da Faculdade de Medicina de Botucatu, especializada no apoio a estudos epidemiológicos.

Os instrumentos para coleta e registro dos dados foram construídos pelos pesquisadores e testados por estudo piloto, visando ajustar as questões que poderiam apresentar possíveis dificuldades. As máscaras para digitação dos dados foram construídas no software Epi Info versão $7.0 \AA$, previamente testadas. Ressalta-se que os 12 binômios participantes do estudo piloto não compuseram a amostra deste estudo, pois foram necessários ajustes no instrumento de coleta de dados, a fim de que a pesquisa pudesse ser iniciada efetivamente.
As variáveis (covariáveis) de caracterização do binômio foram: idade materna menor que 20 anos (sim/não); escolaridade materna menor que oito anos (sim/não); renda per capita menor ou igual a um salário mínimo (sim/não); cor materna não branca (sim/não); mãe não vive com companheiro (sim/não); mãe não exerce trabalho remunerado (sim/não); gestação não planejada (sim/não); parto não realizado no Sistema Único de Saúde (sim/ não); idade gestacional no parto igual ou inferior a 38 semanas (sim/não).

Quanto aos desfechos, foram estudados: interrupção do aleitamento materno exclusivo (AME) antes do sexto mês de vida (não/sim); infecção respiratória: asma, bronquite, bronquiolite, pneumonia e/ou outras infecções respiratórias no primeiro ano de vida (sim/não); atopias: rinite, rinoconjuntivite, conjuntivite, dermatite, alergia alimentar e/ou outras atopias no primeiro ano de vida (sim/não); índice de massa corpórea (IMC) por idade: sobrepeso e obesidade no $12 .{ }^{\circ}$ mês de vida (sim/não).

A variável exposição foi tipo de parto (parto vaginal e cesárea eletiva).

Para identificação da renda per capita familiar, indagouse sobre o rendimento da família em reais e dividiu-se pelo número de pessoas dependentes dessa renda; em seguida, considerando-se o valor do salário mínimo à época da coleta de dados $(\mathrm{R} \$ 788,00)$, classificou-se a renda per capita familiar em salários mínimos.

O estado nutricional foi classificado de acordo com o IMC, segundo a idade. Em relação ao cálculo do IMC e posterior classificação do escore $z$, os dados de peso e estatura dos bebês foram submetidos a análises de consistência de IMC/ idade utilizando-se o programa AnthroPlus ${ }^{\circledR}$. Os pontos de corte para a classificação do bebê quanto ao IMC seguiram as recomendações da Organização Mundial de Saúde: magreza acentuada ( $<$ Escore-z -3 ); magreza ( $>$ Escore-z -3 e $<$ Escore-z -2); eutrofia ( $>$ Escore- $z-2$ e $<$ Escore- $z+1$ ); risco de sobrepeso ( $>$ Escore-z +1 e $<$ Escore-z +2 ); sobrepeso ( $>$ Escore-z +2 e $<$ Escore- $z+3$ ); e obesidade ( $>$ Escore- $z+3) .{ }^{20}$

A associação do tipo de parto com interrupção precoce do AME, infecção respiratória e atopias no primeiro ano de vida e sobrepeso/obesidade aos 12 meses foi analisada, ajustando modelos de regressão múltipla semi-paramétricos de Cox, incluindo, no componente determinístico desses modelos, as variáveis que, na exploração bivariada, apresentaram associação estatística com $p<0,20$. Utilizou-se para as análises o software Statistical Package for the Social Sciences (SPSS) versão 21.0.

A integridade das entrevistas foi verificada em amostra aleatória de $5 \%$ dos participantes, mediante reentrevistas realizadas por telefone pela supervisora de campo. Os bancos de dados foram checados, as inconsistências verificadas nos questionários e, em seguida, realizaram-se as correções nos bancos sempre que pertinente.

O presente projeto foi submetido ao Comitê de Ética em Pesquisa local e aprovado para execução, mediante Parecer n. - 893.396. As mulheres que concordaram em participar do estudo assinaram Termo de Consentimento Livre e Esclarecido. 


\section{RESULTADOS}

Do total de 499 mães/bebês que compuseram a amostra deste estudo, 260 mães (52,1\%) tiveram parto vaginal e 239 $(47,9 \%)$ foram submetidas à cesárea eletiva.

A Tabela 1 apresenta as características sociodemográficas e gestacionais das mulheres e ocorrência dos desfechos em estudo.

Sobre as características maternas em função do tipo de parto, observa-se maior prevalência de cesárea eletiva entre as mulheres cuja gestação foi planejada e com parto não realizado no SUS. Quanto aos bebês, tinham, com maior frequência, idade gestacional ao nascer igual ou inferior a 38 semanas (Tabela 1).

A proporção de lactentes que receberam AME até o sexto mês de vida foi maior entre os nascidos de parto vaginal. Quanto àqueles que tiveram infecção respiratória, atopia e alteração de crescimento (sobrepeso/obesidade) no primeiro ano de vida, a ocorrência foi semelhante entre os dois grupos (Tabela 1).

A seguir, apresentam-se as análises para verificar o efeito da cesárea eletiva e a influência das covariáveis sobre os desfechos selecionados no primeiro ano de vida.

A Tabela 2 trata da interrupção precoce do AME.

O modelo final foi ajustado para as seguintes covariáveis: não exerce trabalho remunerado e parto não SUS $(p<0,20)$. Após ajuste, a interrupção do AME antes do sexto mês de vida não se associou à cesárea eletiva: pvalor $=0,473$; $R R=1,09$; IC95\%= 0,86-1,39 (Tabela 2).

A Tabela 3 trata da ocorrência de infecção respiratória na população estudada.

Após ajustar o modelo para as covariáveis - escolaridade, não vive com companheiro e gestação não planejada $(p<0,20)$ -, infecção respiratória no primeiro ano de vida não foi associada à cesárea eletiva: pvalor=0,930; RR=1,01; IC95\%=0,73-1,41 (Tabela 3).

A Tabela 4 é relativa à ocorrência de sobrepeso/obesidade no $12 .{ }^{\circ}$ mês de vida.

Ao investigar a associação entre sobrepeso/obesidade no 12. ำ mês de vida e cesárea eletiva, após ajuste das covariáveis - idade menor ou igual a 19 anos, não vive com companheiro, renda menor ou igual a um salário mínimo e parto não SUS $(p<0,20)-$, sobrepeso/obesidade não se associou à cesárea eletiva: pvalor=0,564; RR=1,15; IC95\%=0,71-1,84 (Tabela 4).

Com relação às atopias no primeiro ano de vida, nenhuma associação significativa foi constatada (dados não mostrados em tabela).

\section{DISCUSSÃO}

O presente estudo permitiu verificar especificamente o efeito da cesárea eletiva sobre desfechos do primeiro ano de vida; aspecto relevante, tendo em vista que a literatura científica, em geral, não diferencia cesárea eletiva e indicada. Dentre os desfechos estudados, não houve associação ao tipo de parto. Assim, nascer de cesárea eletiva, comparado ao nascimento por parto vaginal, não exerceu, neste estudo, influência sobre as variáveis: interrupção do AME
Tabela 1. Descrição das características sociodemográficas e gestacionais maternas por tipo de parto e desfechos em estudo. Botucatu, 2015-2017.

\begin{tabular}{|c|c|c|c|c|}
\hline \multirow[t]{2}{*}{ Variáveis } & \multicolumn{2}{|c|}{$\begin{array}{l}\text { Parto vaginal } \\
\qquad(n=260)\end{array}$} & \multicolumn{2}{|c|}{$\begin{array}{c}\text { Cesárea } \\
\text { eletiva }(n=239)\end{array}$} \\
\hline & $n$ & $\%$ & $n$ & $\%$ \\
\hline \multicolumn{5}{|l|}{ Idade (anos) } \\
\hline$\leq 19$ & 49 & 18,8 & 28 & 11,7 \\
\hline$\geq 20$ & 211 & 81,2 & 211 & 88,3 \\
\hline \multicolumn{5}{|c|}{ Escolaridade (anos de aprovação) } \\
\hline$\leq 7$ & 32 & 12,3 & 13 & 5,4 \\
\hline$\geq 8$ & 288 & 87,7 & 266 & 94,6 \\
\hline \multicolumn{5}{|l|}{ Cor } \\
\hline Não branca & 106 & 40,7 & 80 & 33,4 \\
\hline Branca & 154 & 59,3 & 159 & 66,6 \\
\hline \multicolumn{5}{|l|}{ Vive com companheiro } \\
\hline Sim & 219 & 84,2 & 219 & 91,6 \\
\hline Não & 41 & 15,8 & 20 & 8,4 \\
\hline \multicolumn{5}{|l|}{ Trabalho remunerado } \\
\hline Sim & 132 & 50,7 & 162 & 67,8 \\
\hline Não & 128 & 49,3 & 77 & 32,2 \\
\hline \multicolumn{5}{|l|}{ Renda familiar per capita } \\
\hline s um salário mínimo & 239 & 91,9 & 155 & 64,8 \\
\hline > um salário mínimo & 21 & 8,1 & 84 & 35,2 \\
\hline \multicolumn{5}{|l|}{ Gestação planejada } \\
\hline Sim & 111 & 42,7 & 133 & 55,6 \\
\hline Não & 149 & 57,3 & 106 & 44,4 \\
\hline \multicolumn{5}{|l|}{ Parto fora do SUS } \\
\hline Sim & 23 & 8,8 & 142 & 59,4 \\
\hline Não & 237 & 91,2 & 97 & 40,6 \\
\hline \multicolumn{5}{|c|}{ Idade gestacional no parto } \\
\hline$\leq 38$ semanas & 78 & 30,0 & 122 & 51,0 \\
\hline$\geq 39$ semanas & 182 & 70,0 & 117 & 49,0 \\
\hline \multicolumn{5}{|c|}{$\begin{array}{l}\text { Aleitamento materno } \\
\text { exclusivo no sexto mês de } \\
\text { vida }\end{array}$} \\
\hline Sim & 78 & 30,0 & 47 & 19,7 \\
\hline Não & 182 & 70,0 & 192 & 80,3 \\
\hline \multicolumn{5}{|l|}{$\begin{array}{l}\text { Infecção respiratória no } \\
\text { primeiro ano de vida }\end{array}$} \\
\hline Sim & 80 & 30,8 & 69 & 28,9 \\
\hline Não & 180 & 69,2 & 170 & 71,1 \\
\hline \multicolumn{5}{|c|}{$\begin{array}{l}\text { Atopia no primeiro ano de } \\
\text { vida }\end{array}$} \\
\hline Sim & 65 & 25,0 & 58 & 24,3 \\
\hline Não & 195 & 75,0 & 181 & 75,7 \\
\hline \multicolumn{5}{|c|}{$\begin{array}{l}\text { Sobrepeso/Obesidade no } \\
12.0 \text { mês de vida }(n=418)\end{array}$} \\
\hline Sim & 32 & 14,8 & 30 & 14,9 \\
\hline Não & 184 & 85,2 & 172 & 85,1 \\
\hline
\end{tabular}


Tabela 2. Associação entre cesárea eletiva e interrupção precoce do aleitamento materno exclusivo, a partir de regressão de Cox bruta e ajustada. Botucatu, 2015-2017.

\begin{tabular}{|c|c|c|c|c|c|c|c|}
\hline \multirow{3}{*}{$\begin{array}{l}\text { Variável } \\
\text { Idade } \leq 19 \text { anos }\end{array}$} & \multicolumn{3}{|c|}{ Análise bruta } & \multirow{3}{*}{$\begin{array}{l}\text { Pvalor } \\
0,698\end{array}$} & \multicolumn{3}{|c|}{ Análise ajustada } \\
\hline & \multirow{2}{*}{$\begin{array}{l}\mathbf{R R}^{*} \\
0,94\end{array}$} & \multicolumn{2}{|c|}{ IC95\%** } & & \multirow[t]{2}{*}{$\mathbf{R R}^{*}$} & \multirow[t]{2}{*}{ IC95\% } & \multirow[t]{2}{*}{ pvalor } \\
\hline & & 0,71 & 1,26 & & & & \\
\hline Cor não branca & 1,09 & 0,88 & 1,34 & 0,417 & & & \\
\hline Não vive com companheiro & 1,13 & 0,84 & 1,52 & 0,405 & & & \\
\hline Não exerce trabalho remunerado & 0,81 & 0,65 & 1,00 & 0,050 & 0,83 & $0,66-1,04$ & 0,105 \\
\hline Escolaridade $\leq 7$ anos & 0,88 & 0,60 & 1,28 & 0,501 & & & \\
\hline Renda per capita $\leq 1$ salário mínimo & 0,96 & 0,75 & 1,23 & 0,770 & & & \\
\hline Gestação não planejada & 0,98 & 0,80 & 1,19 & 0,826 & & & \\
\hline Parto não SUS & 1,15 & 0,93 & 1,43 & 0,176 & 1,03 & $0,80-1,34$ & 0,786 \\
\hline Idade gestacional ao nascer $\leq 38$ semanas & 1,02 & 0,83 & 1,26 & 0,825 & & & \\
\hline Cesárea eletiva & 1,15 & 0,93 & 1,40 & 0,183 & 1,09 & $0,86-1,39$ & 0,473 \\
\hline
\end{tabular}

*RR= Risco Relativo; ${ }^{*}$ IC= Intervalo de Confiança

Tabela 3. Associação entre cesárea eletiva e infecção respiratória em crianças com até 1 ano de idade, a partir de regressão de Cox bruta e ajustada. Botucatu, 2015-2017.

\begin{tabular}{|c|c|c|c|c|c|c|c|}
\hline \multirow{2}{*}{ Variável } & \multirow{2}{*}{$\mathbf{R R}^{*}$} & \multicolumn{3}{|c|}{ Análise bruta } & \multirow{2}{*}{$\mathbf{R R}^{*}$} & \multicolumn{2}{|c|}{ Análise ajustada } \\
\hline & & & & pvalor & & IC**95\% & pvalor \\
\hline Idade $\leq 19$ anos & 0,85 & 0,53 & 1,36 & 0,498 & & & \\
\hline Cor não branca & 1,16 & 0,84 & 1,61 & 0,355 & & & \\
\hline Não vive com companheiro & 1,38 & 0,89 & 2,13 & 0,150 & 1,20 & $0,76-1,90$ & 0,417 \\
\hline Não exerce trabalho remunerado & 0,84 & 0,60 & 1,17 & 0,302 & & & \\
\hline Escolaridade $\leq 7$ anos & 1,56 & 0,97 & 2,50 & 0,063 & 1,44 & $0,88-2,33$ & 0,140 \\
\hline Renda per capita $\leq 1$ salário mínimo & 1,42 & 0,82 & 1,92 & 0,283 & & & \\
\hline Gestação não planejada & 1,38 & 0,99 & 1,91 & 0,053 & 1,31 & $0,93-1,83$ & 0,116 \\
\hline Parto não SUS & 1,08 & 0,77 & 1,52 & 0,634 & & & \\
\hline Idade gestacional ao nascer $\leq 38$ semanas & 1,06 & 0,77 & 1,47 & 0,703 & & & \\
\hline Cesárea eletiva & 0,94 & 0,68 & 1,29 & 0,698 & 1,01 & $0,73-1,41$ & 0,930 \\
\hline
\end{tabular}

*RR= Risco Relativo; **IC= Intervalo de Confiança

Tabela 4. Associação entre cesárea eletiva e sobrepeso/obesidade no 12. mês de vida, a partir de regressão de Cox bruta e ajustada. Botucatu, 2015-2017.

\begin{tabular}{|c|c|c|c|c|c|c|c|}
\hline \multirow{2}{*}{ Variável } & \multirow{2}{*}{$\mathbf{R R}^{*}$} & \multicolumn{3}{|c|}{ Análise bruta } & \multirow{2}{*}{$\mathbf{R R}^{*}$} & \multicolumn{2}{|c|}{ Análise ajustada } \\
\hline & & \multicolumn{2}{|c|}{ IC** 95\% } & \multirow{2}{*}{$\begin{array}{c}\text { pvalor } \\
0,124\end{array}$} & & IC**95\% & pvalor \\
\hline Idade $\leq 19$ anos & 0,59 & 0,30 & 1,15 & & 0,60 & $0,31-1,53$ & 0,126 \\
\hline Cor não branca & 0,81 & 0,54 & 1,24 & 0,343 & & & \\
\hline Não vive com companheiro & 1,74 & 0,97 & 3,13 & 0,063 & 1,69 & $0,96-1,98$ & 0,068 \\
\hline Não exerce trabalho remunerado & 0,93 & 0,60 & 1,44 & 0,746 & & & \\
\hline Escolaridade $\leq 7$ anos & 0,48 & 0,75 & 3,42 & 0,487 & & & \\
\hline Renda per capita $\leq 1$ salário mínimo & 1,54 & 0,87 & 2,71 & 0,136 & 1,48 & $0,87-2,53$ & 0,144 \\
\hline Gestação não planejada & 1,16 & 0,77 & 1,75 & 0,475 & & & \\
\hline Parto não SUS & 0,56 & 0,88 & 2,75 & 0,121 & 1,55 & $0,94-2,58$ & 0,086 \\
\hline Idade gestacional ao nascer $\leq 38$ semanas & 1,04 & 0,68 & 1,60 & 0,832 & & & \\
\hline Cesárea eletiva & 1,07 & 0,65 & 1,74 & 0,791 & 1,15 & $0,71-1,84$ & 0,564 \\
\hline
\end{tabular}

*RR= Risco Relativo; **IC= Intervalo de Confiança 
antes do 6. mês de vida, infecção respiratória e atopia no primeiro ano e sobrepeso/obesidade no 12. mês de vida.

A associação entre desmame e cesárea tem sido evidenciada. Estudo prospectivo desenvolvido no Canadá apontou que mulheres submetidas à cesárea eletiva são mais propensas ao desmame, nas primeiras 12 semanas após o parto, do que mulheres cujo parto foi vaginal $(\mathrm{OR}=1,61$; IC95\%=1,14-2,26; $p=0,014) .{ }^{7}$ Coorte desenvolvida na China, com o objetivo de determinar os efeitos potenciais da cesárea - sem distinção entre eletiva e indicada - sobre a prática e duração do aleitamento materno, também revelou que lactentes nascidos por cesárea desmamaram antes que bebês nascidos por parto vaginal $(\mathrm{OR}=1,40 ; \mathrm{IC} 95 \%=1,06-1,84 ; \mathrm{p}<0,05){ }^{21}$

Tal associação não foi observada no presente estudo: bebês nascidos por cesárea eletiva não tiveram risco aumentado para interrupção do AME antes do sexto mês de vida quando comparados aos nascidos por parto vaginal. Uma das hipóteses que pode justificar a falta de associação é relacionada às características da população estudada, visto que, em ambos os grupos, mais de $80 \%$ das mulheres tinham idade igual ou superior a 20 anos, nível de escolaridade igual ou superior a 8 anos e viviam com companheiro.

Merece destaque o fato de a proporção desta prática alimentar ter sido maior no grupo de bebês nascidos por parto vaginal, quando comparados aos nascidos por cesárea eletiva, e também a relevância clínica relacionada ao pequeno número de crianças mantidas em AME até sexto mês de vida.

Estudo transversal desenvolvido em hospital credenciado como Amigo da Criança, localizado em uma capital do Norte brasileiro - que buscou identificar fatores associados ao AME - também não encontrou associação significativa com tipo de parto. ${ }^{22}$ Dados da Pesquisa Nacional de Saúde, de 2013, apontam que a prevalência da amamentação exclusiva até o sexto mês de vida foi de $36,6 \%$ no país. ${ }^{23}$ Nos Estados Unidos da América, a situação é ainda mais desfavorável: de acordo com o Centro de Controle e Prevenção de Doenças, a prevalência de lactentes amamentados exclusivamente até o sexto mês de vida, no ano de 2016 , foi $25,4 \% .{ }^{24}$ Percebe-se, dessa forma, que a taxa de aleitamento exclusivo $(25,1 \%)$ da população estudada está aquém da prevalência verificada em âmbito nacional e assemelha-se ao resultado americano.

A Organização Mundial de Saúde, o Ministério da Saúde e a Sociedade Brasileira de Pediatria recomendam que essa prática de AME permaneça durante os primeiros seis meses de vida da criança. ${ }^{25} \mathrm{~A}$ nutrição complementar deve ser iniciada após esse período, em conjunto com a amamentação, e manter-se, preferencialmente, até os dois anos de vida, pelo fato de revelarse importante para saúde física e psíquica do binômio. ${ }^{26}$

Pelo exposto, fica evidente a necessidade de ações educativas específicas voltadas às mães - independentemente do tipo de parto -, a fim de estimular o aleitamento materno, intervenções essas que devem se fazer presentes desde o pré-natal, abordando, dentre outras questões, o plano de parto das gestantes e as consequências destes para o binômio.

Sobre a inexistência de associações significativas para os desfechos infecções respiratórias e atopias, as seguintes hipóteses explicativas podem ser levantadas: o seguimento no primeiro ano de vida não foi suficiente para identificar esses desfechos, visto que os estudos que encontraram tal associação as investigaram na infância e em crianças maiores de 6 anos. ${ }^{10-12}$ Estudo que objetivou verificar a associação entre cesárea e asma em crianças de 2 anos ratifica essa afirmativa, pois ressalta a dificuldade deste tipo de diagnóstico em crianças até os 2 anos de idade. ${ }^{8}$ Outra hipótese está relacionada à não identificação, neste estudo, se a cesárea ocorreu antes ou após o início do trabalho de parto, já que a literatura revela associação mais forte entre desenvolvimento de asma em crianças nascidas de cesárea antes da rotura da membrana amniótica. ${ }^{11}$

Também, não se encontrou associação entre cesárea eletiva comparada ao parto vaginal e a ocorrência de sobrepeso/ obesidade e, outra vez, a explicação pode estar no fato de o seguimento realizado - um ano - ter sido insuficiente para identificar alterações de crescimento relacionadas aos tipos de parto, visto que estudos publicados trazem essa associação a médio e longo prazos, especialmente na primeira infância e adolescência. ${ }^{14,15}$

\section{CONCLUSÃO E IMPLICAÇÕES PARA A PRÁTICA}

Este estudo admite a conclusão de que a cesárea eletiva, na população estudada, não exerceu efeito sobre a interrupção do AME antes do 6. mês de vida, ocorrência de infecção respiratória e atopia no primeiro ano e sobrepeso/obesidade no 12. ${ }^{\circ}$ mês de vida. Assim, refuta-se a hipótese apresentada nesta pesquisa.

Como limitações do estudo, os autores destacam que os dados refletem as condições sociodemográficas e de saúde de município do interior paulista. Por isso, a generalização para outras populações deve considerar as particularidades de uma cidade de médio porte. Alguns potenciais confundidores não explorados podem ter impacto sobre as análises, como tempo para início do aleitamento materno ou se houve incentivo ao aleitamento materno precoce, tipo e momento em que foram introduzidos os alimentos, e o fato de não ter sido obtido dado sobre o momento em que a cesárea eletiva ocorreu; ou seja, antes ou após início do trabalho de parto.

Pelos resultados encontrados, sugere-se que a equipe de saúde apoie as mães - independente do tipo de parto, desde o pré-natal -, com o intuito de aumentar sua confiança e empenho em relação à amamentação, buscando prevenir, dessa forma, que eventos deletérios aconteçam. Sugere-se, também, que novos estudos sejam desenvolvidos, com maior tempo de seguimento e identificação dos casos em que a cesárea eletiva foi realizada após o início do trabalho de parto, para melhor compreensão das relações investigadas neste estudo.

\section{FINANCIAMENTO}

Fundação de Amparo à Pesquisa do Estado de São Paulo (FAPESP - auxílio a projeto de pesquisa - Processo n.으 2015/03256-1), concedido ao projeto de pesquisa "Saúde da criança no primeiro ano de vida: estudo de coorte prospectiva no interior paulista", coordenado pela pesquisadora Cristina 
Maria Garcia de Lima Parada. O presente trabalho foi realizado com apoio da Coordenação de Aperfeiçoamento de Pessoal de Nível Superior - Brasil (CAPES) - Código de Financiamento 001 (concessão de bolsa de doutorado para Anna Paula Ferrari).

\section{CONTRIBUIÇÕES DOS AUTORES}

Desenho do estudo: Anna Paula Ferrari.

Coleta de dados: Anna Paula Ferrari. Maria Cristina Heinzle

da Silva Machado.

Análise de dados: Anna Paula Ferrari. Maria Cristina Heinzle da Silva Machado. Vera Lúcia Pamplona Tonete. Cristina Maria Garcia de Lima Parada.

Interpretação dos resultados: Anna Paula Ferrari. Vera Lúcia

Pamplona Tonete. Cristina Maria Garcia de Lima Parada.

Redação e revisão crítica do manuscrito: Anna Paula Ferrari.

Maria Cristina Heinzle da Silva Machado. Vera Lúcia Pamplona Tonete: Cristina Maria Garcia de Lima Parada.

Aprovação da versão final do artigo: Anna Paula Ferrari. Maria Cristina Heinzle da Silva Machado. Vera Lúcia Pamplona Tonete. Cristina Maria Garcia de Lima Parada.

Responsabilidade por todos os aspectos do conteúdo e a integridade do artigo publicado: Anna Paula Ferrari. Maria Cristina Heinzle da Silva Machado. Vera Lúcia Pamplona Tonete. Cristina Maria Garcia de Lima Parada.

\section{EDITOR ASSOCIADO}

Maria Catarina Salvador da Motta.

\section{REFERÊNCIAS}

1. Arruda GT, Barreto SC, Morin VL, Petter GN, Braz MM, Pivetta HMF. Is there a relation between mode of delivery and breastfeeding in the first year of life? Rev Bras Promoç Saude. 2018;31(2):1-7. http://dx.doi. org/10.5020/18061230.2018.7321.

2. Ferrari AP, Carvalhaes MABL, Parada CMGL. Association between prenatal and parturition in the supplementary health network and elective cesarean section. Rev Bras Epidemiol. 2016;19(1):75-88. http://dx.doi. org/10.1590/1980-5497201600010007.

3. Oliveira LL, Gonçalves AC, Costa JSD, Bonilha ALL. Maternal and neonatal factors related to prematurity. Rev Esc Enferm USP. 2016;50(3):382-9. http://dx.doi.org/10.1590/S0080-623420160000400002.

4. Guasch XD, Reymundo MG, Suazo JAH, Aguilar MJC, Galiana GG, Jiménez Moya A. Prematuridad tardía: una población de riesgo. Clin Invest Ginecol Obstet. 2018;45(1):17-23. http://dx.doi.org/10.1016/j. gine.2017.12.001.

5. Vinding RK, Sejersen TS, Chawes BL, Bonnelykke K, Buhl T, Bisgaard $\mathrm{H}$ et al. Cesarean delivery and body mass index 6 months and into childhood. Pediatrcs. 2017;139(6):e20164066. http://dx.doi.org/10.1542/ peds.2016-4066.

6. Gabet S, Just J, Couderc R, Seta N, Momas I. Allergic sensitisation in early childhood: patterns and related factors in Paris birth cohort. Int $\mathrm{J}$ Hyg Environ Health. 2016;219(8):792-800. http://dx.doi.org/10.1016/j. ijheh.2016.09.001.

7. Hobbs AJ, Mannion CA, McDonald SW, Brockway M, Tough SC. The impact of caesarean section on breastfeeding initiation, duration and difficulties in the first four months postpartum. BMC Pregnancy Childbirth. 2016;16(1):90. http://dx.doi.org/10.1186/s12884-016-0876-1.

8. Rodrigues SML, Silva PMM. Parto eutócico versus cesariana eletiva e a incidência de patologias na criança até aos 2 anos. Rev Enf Ref 2017;serIV (15):83-90. http://dx.doi.org/10.12707/RIV17051.
9. Prior E, Santhakumaran S, Gale C, Philipps LH, Modi N, Hyde MJ Breastfeeding after cesarean delivery: a systematic review and metaanalysis of world literature. Am J Clin Nutr. 2012;95(5):1113-11135. http://dx.doi.org/10.3945/ajcn.111.030254.

10. Merenstein DJ, Gatti ME, Mays DM. The association of mode of delivery and common childhood illnesses. Clin Pediatr. 2011;50(11):1024-30. http://dx.doi.org/10.1177/0009922811410875.

11. Sevelsted A, Stokholm J, Bisgaard $\mathrm{H}$. Risk of asthma from cesarean delivery depends on membrane rupture. J Pediatr. 2016;171:38-42. http://dx.doi.org/10.1016/j.jpeds.2015.12.066.

12. Papathoma E, Triga M, Fouzas S, Dimitriou G. Cesarean section delivery and development of food allergy and atopic dermatitis in childhood. Pediat Allergy Immunol. 2016;27(4):419-24. http://dx.doi.org/10.1111/pai.12552.

13. Tun HM, Bridgman SL, Chari R, Field CJ, Guttman DS, Becker AL et al Roles of birth mode and infant gut microbiota in intergenerational transmission of overweight and obesity from mother to offspring JAMA Pediatr. 2018;172(4):368-77. http://dx.doi.org/10.1001/ jamapediatrics.2017.5535.

14. Ramos CV, Dumith SC, César JA. Prevalence and factors associated with stunting and excess weight in children aged 0-5 years from the Brazilian semi-arid region. J Pediatr. 2015;91(2):175-82. http://dx.doi. org/10.1016/j.jped.2014.07.005.

15. Gomes AT, Novaes TG, Silveira KC, Souza CL, Lamounier JA, Netto MP et al. Excess weight and factors associated in preschool of southwest of Bahia. Rev Bras Saúde Mater Infant. 2017;17(2):375-83. http://dx.doi. org/10.1590/1806-93042017000200009.

16. Nunes RD, Traebert E, Semann M, Traebert J. Cesarean birth and the nutritional development in childhood: results from a cohort in Southern Brazil. RBONE [Internet]. 2019; [citado 2019 jan 4];13(77):40-5. Disponível em: http://www.rbone.com.br/index.php/rbone/article/view/872

17. Instituto Brasileiro de Geografia e Estatística, Diretoria de Pesquisas, Coordenação de População e Indicadores Sociais. Estimativas da população residente com data de referência $1 \stackrel{\circ}{ }$. de julho de 2018 [Internet]. 2018 [citado 2019 jan 4]. Disponível em: https://cidades. ibge.gov.br/brasil/sp/botucatu/panorama

18. Oliveira RLA, Fonseca CRB, Carvalhaes MABL, Parada CMGL. Avaliação da atenção pré-natal na perspectiva dos diferentes modelos na atenção primária. Rev Lat Am Enfermagem [Internet]. 2013; [citado 2019 jan 4];21(2):[aprox. 8 telas]. Disponível em: https://www.scielo.br/ pdf/rlae/v21n2/pt_0104-1169-rlae-21-02-0546.pdf

19. Ministério da Saúde (BR), Comissão Nacional de Incorporação de Tecnologias no SUS. Diretrizes de atenção à gestante: a operação cesariana. Brasília: Ministério da Saúde; 2016.

20. World Health Organization. Child growth standards [Internet]. 2009 [citado 2018 set 7]. Disponível em: https://www.who.int/childgrowth/ standards/chts_bfa_girls_z/en/

21. Chen C, Yan Y, Gao X, Xiang S, He Q, Zeng G et al. Effects of cesarean delivery on breastfeeding practices and duration: a prospective cohort study. J Hum Lact. 2018;34(3):526-34. http://dx.doi. org/10.1177/0890334417741434.

22. Margotti E, Margotti W. Fatores relacionados ao aleitamento materno exclusivo em bebês nascidos em hospital amigo da criança em uma capital do Norte brasileiro. Saúde Debate. 2017:41(114):860-71. http:// dx.doi.org/10.1590/0103-1104201711415.

23. Boccolini CS, Boccolini PMM, Monteiro FR, Venâncio SI, Giugliani ERJ. Breastfeeding indicators trends in Brazil for three decades. Rev Saude Publica. 2017;51:108. http://dx.doi.org/10.11606/S15188787.2017051000029.

24. Centers for Disease Control and Prevention. Breastfeeding rates [Internet]. 2018 [citado $2018 \mathrm{dez}$ 10]. Disponível em: https://nccd. cdc.gov/dnpao dtm/rdPage.aspx?rdReport=DNPAO DTM. ExploreByTopic\&is $I$ Class $=B F \&$ islTopic $=B F 1 \& g 0=G O$

25. Severiano AAO, Dantas DS, Oliveira VLC, Lopes JM, Souza DE, Magalhães AG. Association between breastfeeding, obstetric factors and child development in northeast Brazil. J Hum Growth Dev. 2017;27(2):15865. http://dx.doi.org/10.7322/jhgd.114483.

26. Morais MB, Cardoso AL, Lazarini T, Mosquera BEM, Mallozi MC. Habits and attitudes of mothers of infants in relation to breastfeeding and artificial feeding in 11 brazilian cities. Rev Paul Pediatr. 2017;35(1):39-45. http:// dx.doi.org/10.1590/1984-0462/;2017;35;1;00014 